\title{
A Farewell to the Encephalization Quotient: A New Brain Size Measure for Comparative Primate Cognition
}

\author{
Carel P. van Schaik ${ }^{a, b}$ Zegni Triki ${ }^{c, d}$ Redouan Bshary ${ }^{c}$ Sandra A. Heldstab ${ }^{a}$ \\ a Department of Anthropology and Anthropological Museum, University of Zurich, Zurich, Switzerland; \\ ${ }^{b}$ Department of Evolutionary Biology and Environmental Science, University of Zurich, Zurich, Switzerland; \\ 'Behavioral Ecology Laboratory, Faculty of Science, University of Neuchâtel, Neuchâtel, Switzerland; ${ }^{\mathrm{d}}$ Institute of \\ Zoology, Stockholm University, Stockholm, Sweden
}

\section{Keywords}

Encephalization quotient · Cognitive equivalence ·

Intelligence $\cdot$ Mammals $\cdot$ Hominins

\begin{abstract}
Both absolute and relative brain sizes vary greatly among and within the major vertebrate lineages. Scientists have long debated how larger brains in primates and hominins translate into greater cognitive performance, and in particular how to control for the relationship between the noncognitive functions of the brain and body size. One solution to this problem is to establish the slope of cognitive equivalence, i.e., the line connecting organisms with an identical bauplan but different body sizes. The original approach to estimate this slope through intraspecific regressions was abandoned after it became clear that it generated slopes that were too low by an unknown margin due to estimation error. Here, we revisit this method. We control for the error problem by focusing on highly dimorphic primate species with large sample sizes and fitting a line through the mean values for adult females and males. We obtain the best estimate for the slope of circa 0.27 , a value much lower than those constructed using all mammal species and close to the
\end{abstract}

karger@karger.com www.karger.com/bbe

Karger $\stackrel{\text { ' }}{5}$
(C) 2021 The Author(s)

Published by S. Karger AG, Basel

This is an Open Access article licensed under the Creative Common Attribution-NonCommercial-4.0 International License (CC BY-NC) (http://www.karger.com/Services/OpenAccessLicense), applicable to the online version of the article only. Usage and distribution for commercial purposes requires written permission. value expected based on the genetic correlation between brain size and body size. We also find that the estimate of cognitive brain size based on cognitive equivalence fits empirical cognitive studies better than the encephalization quotient, which should therefore be avoided in future studies on primates and presumably mammals and birds in general. The use of residuals from the line of cognitive equivalence may change conclusions concerning the cognitive abilities of extant and extinct primate species, including hominins.

(c) 2021 The Author(s)

Published by S. Karger AG, Basel

\section{Introduction}

Although recent ecological approaches to comparative cognition have focused on linking performance in specific cognitive tasks to specific brain regions [e.g., Healy and Krebs, 1996], traditionally comparative cognition has relied on a presumed link between some summary measure of cognitive performance and total brain size [Jerison, 1973]. Scholars have therefore long been searching for a neuroanatomical measure of overall cognitive ability, both to compare living species and to estimate the 
cognitive abilities of extinct ones relative to their extant relatives. The most intuitive measure is a species' brain size (or, especially for extinct species, the highly similar cranial capacity) [Isler et al., 2008]. However, because brains also control numerous noncognitive somatic functions, most researchers have agreed that it cannot be used without controlling for these somatic functions.

Building on a century-long tradition, Jerison [1973] distinguished between the somatic and the cognitive brain functions and proposed that we can estimate the portion of the brain dedicated to somatic functions, and thus by subtraction arrive at the size of the cognitive portion. Jerison [1973] fitted regression equations to the brain size-body size data of all available species in many different mammalian lineages. Because this produced a regression slope close to 0.67 in a log-log plot, he interpreted this as reflecting a fundamental physiological regularity linking brain size to the body's surface area and thus the amount of proprioceptive inputs. This slope could therefore serve as the expected value of the somatic portion of the brain, and the deviation from the regression line as the estimate of the cognitive portion, i.e., cognitive brain size. Jerison [1973] then proposed the encephalization quotient (EQ), the ratio of a species' actual brain size to its predicted brain size based on the cladespecific brain-body regression line, to capture its relative cognitive performance. The EQ has become a commonly used estimate of the cognitive abilities or intelligence of animal species. Other researchers have argued the slope is actually somewhat higher, linking it to metabolic turnover instead and thus suggesting a slope of 0.75 [Martin, 1981; Armstrong, 1983], but they have not questioned the fundamental EQ approach.

Most research on basic relationships between brain size, body size, and cognitive performance has been conducted on mammals, and in particular primates. This research has produced various lines of evidence arguing against the EQ approach. First, its use assumes that there is no correlation between body size and cognitive performance, but in practice there is a negative correlation between EQ and body size within mammalian orders (see below). We should therefore see that bigger mammal species show a poorer cognitive performance than smaller relatives in the same order. However, the opposite appears to be the case [Rensch, 1973], suggesting a lower value of the slope. Second, a lower slope would also be expected given the combined effect of 2 persistent macroevolutionary trends, i.e., (1) brains have increased relative to body size, a phenomenon we can call the Lartet-Marsh rule [Jerison, 1973], and (2) body size has increased as well, a phenomenon known as the Cope rule [Alroy, 1998]. As a result, more recently evolved lineages will tend to have both larger body sizes and larger brain sizes [e.g., Halley and Deacon, 2017], which artificially inflates the slope of the regression through the total sample. Third, different mammalian lineages unexpectedly show different regression slopes of brain size on body size [Martin and Harvey, 1985].

These inconsistencies reveal the fundamental flaw in the EQ approach [Deacon, 1990; Striedter, 2005], it assumes that one process (be it proprioception or metabolic turnover) predominates in determining brain size to the extent that cognitive functions produce only minor deviations, so we can estimate their strength by taking ratios or residuals. Instead, we may expect a variety of processes to affect brain size, with some of them lineage specific and each potentially varying in strength across lineages. Nonetheless, EQs continue to be used to compare mammalian taxa [e.g., Boddy et al., 2012], especially extinct ones [e.g., Grabowski et al. 2016; Benoit et al., 2019].

Although EQ approaches are inadequate, no universally accepted alternative has emerged. A major reason is that the notion of overall cognitive ability or performance remained vague, making it harder to produce an alternative measure. In fact, modern behavioral ecology generally assumes that animals have bundles of domain-specific cognitive adaptations [Shettleworth, 2010]. Consequently, "overall" cognitive performance does not exist and overall brain size does not necessarily provide any useful information when it comes to understanding the animal's ecological niche or social organization. Instead, researchers relate the relative size of specific brain regions to specific cognitive challenges to look for patterns at that level in large comparative surveys. Examples include seed caching and recovery [Healy and Krebs, 1996; Garamszegi and Eens, 2004] or the incidence of feeding innovations [Timmermans et al., 2000] in birds.

Recently, however, brain size has regained standing as a relevant variable. First, along with the unquestioned selective increase or decrease in certain brain parts in response to specific pressures [Barton and Harvey, 2000], mammalian brains are also organized in fundamentally similar ways across animals of varying sizes, even across lineages, which produces predictable allometric relationships among the sizes of the various brain regions [Finlay and Darlington 1995; Finlay et al., 2001]. Indeed, many cognitive activities correspond to concerted activity waves throughout the brain rather than being tied to one particular region [Park and Friston, 2013], suggesting that a 
decrease or increase in one particular region under selection will affect the sizes of other parts and thus also the size of the whole brain [Barton, 2006]. Second, an important consequence of cognitive abilities that tie together many brain regions is domain-general intelligence [Burkart et al., 2017]. This concept has historically been applied mainly to humans, and many, implicitly or explicitly, still consider it to be uniquely human. There is now mounting evidence, however, that, at least among various mammals [Burkart et al., 2017] and birds [Ashton et al., 2018], domain-general intelligence can be recognized within species, and that different species vary considerably in the strength of this domain-general ability [Deaner et al., 2006; Reader et al., 2011]. The latter finding allows empirical tests of the predictive value of EQ or conceptually similar residuals. Broad comparative analyses of estimated domain-general cognitive abilities have shown that EQ is a poor predictor of these abilities [Deaner et al., 2007; Reader et al., 2011], and more limited analyses have reached the same conclusion [Alba, 2010; Rumbaugh et al., 1996]. Moreover, as noted above, most large-bodied species have greater cognitive abilities than expected based on their EQ values [Rensch, 1973], which tend to decrease with body size within orders. In conclusion, these more direct, cognition-based tests of EQ-based approaches confirm that they do not predict cognitive abilities.

\section{Non-EQ Approaches}

These conceptual and empirical problems with EQ measures indicate that we need to control for body size in a different way. Some scholars have suggested that no control is needed at all [e.g., Rensch, 1973; Byrne, 1995; Striedter, 2005], but most accept that regulation of somatic functions requires at least some brain resources that are not also available for cognitive functions. Two main alternatives have been proposed. The first set of techniques is similar to the EQ approach, as it relies on broad interspecific comparisons. Portmann [1946, 1947] proposed that Galliformes are the most primitive birds and deviations from their interspecific regression equation should be used to estimate encephalization. Stephan [1960] proposed the same procedure for mammals, using the "basal insectivores" as the baseline. This produces a "progression index" for each species. However, due to various conceptual and statistical problems [Deacon, 1990] it has found little application. This index also predicts primate cognitive performance only marginally better than EQ and far worse than brain size per se [Gibson et al., 2001; Deaner et al., 2007], although it is unknown how well it does for other mammals or birds.

Cognitive Equivalence in Primates
Another interspecific approach uses ratios of different brain regions, assuming that one, i.e., the numerator, is responsible for the brain's cognitive functions, and the other, i.e., the denominator, is responsible for its somatic functions [Krompecher and Lipák, 1966; Passingham, 1982]. This ratio then estimates the development of cognitive functions relative to expectation. The most popular measure is the neocortex ratio, i.e., the size of the neocortex relative to the rest of the brain [Dunbar, 1992]. However, all ratio measures have the fundamental drawback that they lack a clear neurobiological justification [Deacon, 1990; Deaner et al., 2000; Barton, 2006], and the neocortex ratio was favored simply because it yielded the best correlation with the putative selective pressure [Dunbar, 1992]. Like most other ratios, the neocortex ratio is clearly correlated with both overall brain and body size, as expected based on the fundamental brain allometries [Finlay and Darlington, 2001; Halley and Deacon, 2017]. Thus, its use depends entirely on the goodness of the fit with presumed selective pressures, risking circularity. If this fit varies across taxa, we cannot tell whether a poor fit reflects an imperfect neuroanatomical measure or different selective environments [Stout, 2018]. Moreover, the neocortex ratio does not differentiate between monkeys and apes, which are known to differ in cognitive performance [Gibson et al., 2001]. Also, it does not always predict actual cognitive performance in the primate sample as strongly as other measures when we control for phylogenetic nonindependence [e.g., Deaner et al., 2007]. We will therefore not further consider ratio measures (but see Discussion).

\section{The Slope of Cognitive Equivalence}

The aim of this paper is to revisit the main remaining alternative, i.e., estimating the slope of cognitive equivalence. Its logic works as follows: we assume (1) that the cognitive performance of adult individuals within a species does not depend on their body size and (2) that the intraspecific relationship between body size and brain size among adults estimates the extra amount of brain tissue required to sustain the additional somatic functions in larger individuals because there are no changes in the bauplan or sensory-motor abilities. Therefore, assuming that intraspecific variation in adult size is based on selection on body size alone, the regression of brain size on body size should give us the slope of cognitive equivalence. If analyses conducted on a range of species converge on a similar slope, the cognitive brain size of a species can then be estimated as the residual of the actual brain size of that species from a general line with that slope and some informative intercept. 
This is not a new idea. Many estimates of this slope from samples of conspecific individuals of a known body and brain mass have been made in the past. However, they vary considerably. Summarizing previous work, Pilbeam and Gould [1974] noted values between 0.2 and 0.4. The range for individual mammal species with larger samples was somewhat tighter, with average values between 0.23 and 0.25 [e.g., Hemmer, 1971; Röhrs, 1986] for large intraspecific samples. In large multispecies analyses Martin and Harvey [1985] obtained a median value of 0.14 for primates, and in a large recent analysis of birds and mammals Tsuboi et al. [2018] found a value of 0.15; in both cases the values per species were highly variable. This considerable variability arises because empirical slopes within species are usually underestimated [Pagel and Harvey, 1989] by varying margins. Thus, if we can remove this source of error, this measure's utility could be restored.

The high variability in slope estimates can be explained. The equation for the slope of a regression is [Lande, 1985]:

$$
\operatorname{Slope}(y, x)=\operatorname{Corr}(y, x) \times \frac{\sigma(y)}{\sigma(x)}
$$

where $\operatorname{Corr}(\mathrm{y}, \mathrm{x})$ is the correlation between $\mathrm{x}$ and $\mathrm{y}$, and $\sigma$ is the SD. Because virtually all mammals show determinate growth, there is little variation in body and brain size. As a result, variation due to measurement error can greatly affect the estimated slope of the brain-body relation within a set of adults. Moreover, the error appears to be much larger for body size than for brain size. Brain mass or endocranial volume (our estimate for brain size) is rather constant because during periods of starvation brains continue to receive the same energy flow as during times of plenty, whereas the rest of the body must make do with less. This phenomenon is known as brain sparing [Wells, 2010], resulting in a tiny reduction of brain mass. As a result, the estimate of body size, i.e., body mass, is more variable. Not only does the body lose fat and other tissues during starvation, but it also accumulates fat during times of plenty, whereas the brain does not. The seasonal variation in body mass may therefore greatly exceed the one in brain mass, except for a small number of species with seasonal variation in brain mass [Dechmann et al., 2017]. Among females, body mass also varies across the reproductive cycle, with higher values during pregnancy and lower values toward the end of lactation. Finally, captivity, too, can increase differences in body weight between wild and captive specimens, especially in slow- growing species [e.g., Isler et al., 2008; Leigh 1994]. Due to these various processes, the variance in body mass at a given size can be up to 4 times as high as that in brain mass [Pagel and Harvey, 1989], and the slope will inevitably be underestimated, potentially by up to a factor 2 . To reduce this erroneous reduction in the slope estimate, we should include a greater range of intraspecific body sizes in the analysis, reduce the error in the estimation of each point by taking averages where possible, and use only wild specimens.

One seemingly obvious way to achieve this is to use the means of higher units (species, genera, and families) as data points. This does indeed produce steeper slopes [Martin and Harvey, 1985]. However, while this so-called taxon level effect is partly due to the reduction of noise due to varying body mass, it also reflects the combined Cope-Marsh effects noted above, which will also produce higher slopes for families and orders [Jerison, 1973; Rowe et al., 2011]. Therefore, because we cannot disentangle this Cope-Marsh effect and the estimation error effect, slopes obtained among related species or genera need not reflect cognitive equivalence. However, this slope may still be useful, as it provides a convenient upper limit to the actual slope of cognitive equivalence. The analysis of congeneric slopes by Isler et al. [2008] using independent contrasts yielded a mean slope of 0.41 for primates, suggesting that the true slope lies somewhere between 0.15 and 0.40 .

The best option thus remains to obtain intraspecific slopes that are affected as little as possible by the error problem. We can achieve this by looking for species with a large variation in adult body size. Using data on dog breeds, which show a 30 -fold variation in body size, Bronson [1979] obtained a slope of 0.27 based on averages per breed. This would seem to provide an excellent estimate of the slope of cognitive equivalence but, since dog breeds were produced by artificial selection, we cannot be sure that cognitive equivalence was maintained across the whole size range [cf., Martin and Harvey, 1985].

Here, we consider a variation on this theme. We focus on primates with clear sexual dimorphism in body size so as to minimize error due to the small range in adult body sizes. Second, we take the means of males and females so as to reduce the error in individual data points. And third, we only consider data from wild specimens. We then estimate the slope by fitting a line through the female and male average (which we will refer to as the 2-point slope). We predict that this 2-point slope will be steeper than the slope through all available data points and closer to the true, unbiased value. 
This 2-point slope will be biased when the sexes differ in body composition, in which case body mass is not a good estimate of actual (lean) body size [Schoenemann, 2004]. In humans (not in our sample), men's bodies are far leaner than women's bodies, even among foragers [Wells, 2010]. Obviously, the 2-point slope would then be overestimated. However, such major sex differences in adiposity are not found among nonhuman primates because both arboreality and the high mobility under natural conditions will strongly limit adiposity and thus reduce any such differences [Heldstab et al., 2016; Sterck et al., 2019]. By including only animals taken from the wild, we largely eliminate this possible confounding effect.

The 2-point slope will also be biased when males and females experience differential selection pressures on overall cognitive abilities, in particular due to sexual selection, which by definition may affect the sexes differently. We do not expect this to be important because we know of no studies of primate cognition that needed to control for sex [e.g., Amici et al., 2012; Hopkins et al., 2014; Damerius et al., 2017; see also Arden and Adams, 2016, for dogs]. Likewise, for humans most experts agree that there is no gender difference in intelligence, although some argue for a small difference [Irwing and Lynn, 2005], which may also reflect differential socialization rather than a difference in body size. Moreover, for primates, Lindenfors et al. [2007] found no evidence that sexual selection affects the relative neocortex size, the largest part of primate brains. Thus, this assumption seems warranted. However, for birds, Garamszegi et al. [2005] found a weak positive effect of extra-pair copulation on female brain size. Likewise, Kotrschal et al. [2012] found that males in an Icelandic population of threespined sticklebacks had far larger brains than females, although they could only speculate about the selective agent (perhaps male-only parental care). This effect is less likely in our sample because we focused on highly dimorphic species, thus excluding major variation in the mating system. Nonetheless, to control for the possibility, we will compare species with single-male versus multimale mating. A final possible effect of sexual selection is that there may be an upper limit to the males' ability to maintain cognitive equivalence as body mass dimorphism increases, because females are thought to be at the ecologically optimal size for a given niche. We therefore also examine the importance of dimorphism as a factor in the slope.

Given these various assumptions and our inability to fully test all of them, it is essential to seek external validation. There are 2 ways of doing this. First, we can assess the ecological validity by asking whether the residual brain size values based on the new slope actually predict cognitive abilities across species and do so better than alternative neuroanatomical measures that have been proposed in the past. This test faces a major hurdle in that we do not know whether the relationship between the correct neuroanatomical measures and cognitive performance is linear, more than linear, or less than linear. This problem is exacerbated by the fact that the performance measures are often normalized or even ordinal. However, since the relationship is necessarily monotonic, the correct measure should always preserve the rank order in cognitive performance. Although the different neuroanatomical measures we compare are likely to show high rank correlations among each other, we can nonetheless test their predictive value by assessing the value of the rank correlations with cognitive performance. We found 2 published data sets comparing species' cognitive performance for this analysis. A second way to validate the slope value is to compare it with the strength of genetic correlations between brain size and body size [Lande, 1979]. We will do this in the Discussion, once we have acquired our estimate.

In this study, we therefore first determined the slopes of cognitive equivalence for sexually dimorphic primate species. We used 2 samples (one using conservative and another using more relaxed sample size criteria) and assessed the possible effects of sample sizes, mating system, and sexual dimorphism. Next, we validated the slope we obtained by comparing it with the genetic correlation between brain and body size and by its rank correlation with published measures of species' cognitive abilities. Finally, we made a first, preliminary assessment of the consequences of adopting this new measure of a species' cognitive abilities, using extinct hominins.

\section{Materials and Methods}

We compiled data on the cranial capacity and body mass of primates from the studies by Heldstab et al. [2018, 2019]. We selected species with fairly large sample sizes ( $n \geq 5$ for each sex) to minimize error in estimating the mean body and brain size of males and females. We only included wild-caught animals to avoid captivity effects on body mass (usually fattening), and fully adult individuals, as evinced by the eruption of the third molar. Finally, we set a minimal mass dimorphism at 1.20 because preliminary analysis, as expected, revealed wildly fluctuating (including negative) estimated 2 -point slopes at values close to monomorphism. Overall, we had 18 primate species that met these criteria. The brain-body size slope was then estimated for each species as the slope of the line connecting the male and female average (the 2-point slope), as well as the slope through all of the points. A second primate data set was produced by including all species with at least 10 adult individuals and 
at least 2 of each sex and mass dimorphism of at least 1.20. This data set contained 27 species. We expected more variance in the estimate of the slope. We refer to these 2 data sets as the conservative and relaxed primate data set, respectively.

We examined the bivariate effects of various variables discussed above on the estimated slope: mating system (single male vs. multimale) and female brain size, as well as sexual dimorphism (to assess whether it has a positive effect on estimated slope for which we should control), and sample size (likewise). We also used a model selection approach to identify the best-fitting model.

For the validation part, we used published data sets to capture cognitive performance, i.e., global cognitive performance [Deaner et al., 2007] and the general intelligence factor $g_{s 1}$ from Reader et al. [2011].

The independent measures we used are:

1. Body size ( $\mathrm{P}$, estimated as body mass in $\mathrm{g})$,

2. Brain size (E, estimated as endocranial volume in $\mathrm{mL}$, roughly corresponding to mass in $\mathrm{g}$ [Isler et al. 2008]),

3. Encephalization quotient of Jerison $[1973]\left(\mathrm{E} /\left[0.12 \times \mathrm{P}^{0.667}\right]\right)$, and

4. An estimate of the cognitive brain based on cognitive equivalence $\left(\mathrm{E}-\left[0.065 \times \mathrm{P}^{0.27}\right]\right)$, where the exponent 0.27 is the midpoint value of the empirically obtained intraspecific 2-point slopes in the first set of analyses and the intercept based on one of the smallest-brained mammals, i.e., Sorex minutus, which has a 5-g body mass and a 0.1 -g brain weight [Bauchot and Stephan, 1966]. This measure ensures that virtually all mammals have a positive cognitive brain size.

Note that measure 4 is an absolute measure of the amount of brain tissue available for cognitive tasks, whereas measure 3, i.e., the EQ, is a ratio. Thus, 2 species that differ greatly in body size can have the same EQ, yet the larger of the 2 will have far higher values of measure 4 . Note, too, that measure 4 may be negative for ectothermic vertebrates [cf., Jerison, 1973], and thus may only be intuitive for birds and mammals.

As noted above, we used rank correlations to assess the fit of these measures.

\section{Results}

\section{Estimating the Value of the Slope of Cognitive}

\section{Equivalence}

As expected, the 2-point slopes based on mean values per sex are better than those based on all individual points. Using the conservative primate data set, we found that the slopes using all of the data points (mean $=0.209$ and SEM $=0.020$ ) were on average $30 \%$ less steep than the 2 -point slopes $($ mean $=0.268$ and SEM $=0.020)$. Likewise, the more conservative data set, with at least 5 adult individuals of each sex, gave more reliable slope estimates (lower SEM) than the more relaxed data set, which also varied less with sexual dimorphism (Table 1). Thus, the best estimate of the slope is 0.27 .

The analyses of the possible effect of confounding variables were performed on the conservative primate data
Table 1. Effect of changing the minimal cutoff point of sexual dimorphism for inclusion into the analysis of the 2-point slopes for the conservative and the relaxed primate samples

\begin{tabular}{|c|c|c|c|c|c|c|}
\hline \multirow[t]{2}{*}{$\mathrm{SD} \min$} & \multicolumn{3}{|c|}{ Conservative sample } & \multicolumn{3}{|c|}{ Relaxed sample } \\
\hline & slope & SEM & $\begin{array}{l}\text { species, } \\
n\end{array}$ & slope & SEM & $\begin{array}{l}\text { species, } \\
n\end{array}$ \\
\hline 1.20 & 0.268 & 0.020 & 18 & 0.246 & 0.023 & 27 \\
\hline 1.25 & 0.274 & 0.022 & 16 & 0.262 & 0.022 & 23 \\
\hline 1.30 & 0.258 & 0.017 & 15 & 0.251 & 0.020 & 22 \\
\hline 1.40 & 0.258 & 0.017 & 15 & 0.251 & 0.020 & 22 \\
\hline 1.50 & 0.259 & 0.023 & 8 & 0.262 & 0.027 & 13 \\
\hline
\end{tabular}

SD min, minimal cutoff point of sexual dimorphism.

set only, and thus using only species with sexual dimorphism of $\geq 1.20$, where the standard errors of the estimated slope values had stabilized. Our limited sample size forced us to do bivariate tests. In these tests, we did not need to correct for phylogenetic nonindependence because the Pagel [1992] $\lambda$ values of the slopes were $<0.001$. We found no effect of sample size $(r=-0.041, n=18 ; p=$ 0.87 ) on the value of the 2 -point slope. Likewise, there was no effect of sexual dimorphism $(r=-0.093 ; p=0.71)$ or of mating system (single male vs. multimale; $t_{[16]}=-1.39$; $p=0.18$ ). Moreover, none of the various possible multivariate models showed anywhere near a significant result and multiple models had a close similarity in overall fit (not shown).

\section{Validating the Value of the Slope}

Table 2 provides the values of the Spearman rank correlations for the validation studies for each of the 5 measures used. Overall, as expected because of the need to use ranks only, the results were very close, and the confidence limits for the various measures generally overlapped. Nonetheless, in both cognitive performance measures (data from Deaner et al. [2007] and Reader et al. [2011]), the EQ yielded the lowest correlations, whereas the cognitive brain estimate, absolute brain size, and even body size did about equally well.

\section{Discussion}

\section{Value of the Slope}

We examined the proposition that there is a slope of cognitive equivalence, which predicts the change in brain 
Table 2. Validation analyses with multiple data sets of cognitive performance, using Spearman rank correlations

\begin{tabular}{|c|c|c|c|c|}
\hline Predictor variable & $r_{\mathrm{s}}$ & $p$ value & $95 \% \mathrm{CI}$ & $99 \%$ CI \\
\hline \multicolumn{5}{|c|}{ Deaner et al. [2007] $(n=24)$} \\
\hline $\ln ($ brain size $)$ & 0.811 & $<0.001$ & 0.507 to 0.767 & 0.452 to 0.795 \\
\hline ln (body size) & 0.845 & $<0.001$ & 0.452 to 0.736 & 0.393 to 0.767 \\
\hline Cognitive brain & 0.809 & $<0.001$ & 0.602 to 0.913 & 0.509 to 0.933 \\
\hline Jerison brain & 0.542 & 0.007 & 0.178 to 0.775 & 0.045 to 0.823 \\
\hline \multicolumn{5}{|c|}{ Reader et al. [2011] $(n=28)$} \\
\hline $\ln ($ brain size $)$ & 0.512 & 0.005 & 0.172 to 0.742 & 0.050 to 0.793 \\
\hline ln (body size) & 0.515 & 0.005 & 0.176 to 0.744 & 0.055 to 0.794 \\
\hline Cognitive brain & 0.517 & 0.005 & 0.179 to 0.746 & 0.057 to 0.795 \\
\hline Jerison brain & 0.408 & 0.031 & 0.042 to 0.678 & -0.081 to 0.739 \\
\hline
\end{tabular}

size, and thus cognitive abilities, corresponding to a change in body size in which all of the details of bauplan and sensory-motor abilities are kept constant. Existing estimates of this slope suffer from the problem of measurement error, because mammals generally have only a narrow range of body sizes, whereas their estimates (body mass) can vary widely. We therefore used mean values for males and females in sexually dimorphic species, which have a larger range of body sizes, to estimate the 2-point slope. In general, this approach yielded steeper slopes than regressions through all available data points, confirming the expected reduction in the effect of error on slope estimates and explaining why previous studies often found shallower slopes.

We found no effect of possible confounding variables, such as mating system or dimorphism beyond 1.20. The slope of 0.27 obtained here is the same as the one found for dogs, where breeds vary greatly in size [Bronson, 1979], but, as expected, somewhat higher than the value of 0.23 [Hemmer, 1971] or 0.25 [Röhrs, 1986] found in previous intraspecific mammal studies with large samples that used all individual data rather than estimating 2-point slopes.

\section{Validation of the Slope Estimate}

Based on selection experiments on body size in inbred mice lines, Lande $[1979,1985]$ predicted a 0.36 slope of $\log$ (brain size) on log (body size), which would also hold when body size changes by drift, and thus also when populations contain selectively neutral genetic variation in body size. Slopes observed after selection experiments on body size in rodents yielded values between 0.2 and 0.4 [Riska and Atchley, 1985], but since these should also be affected by the error problem their true value may also be

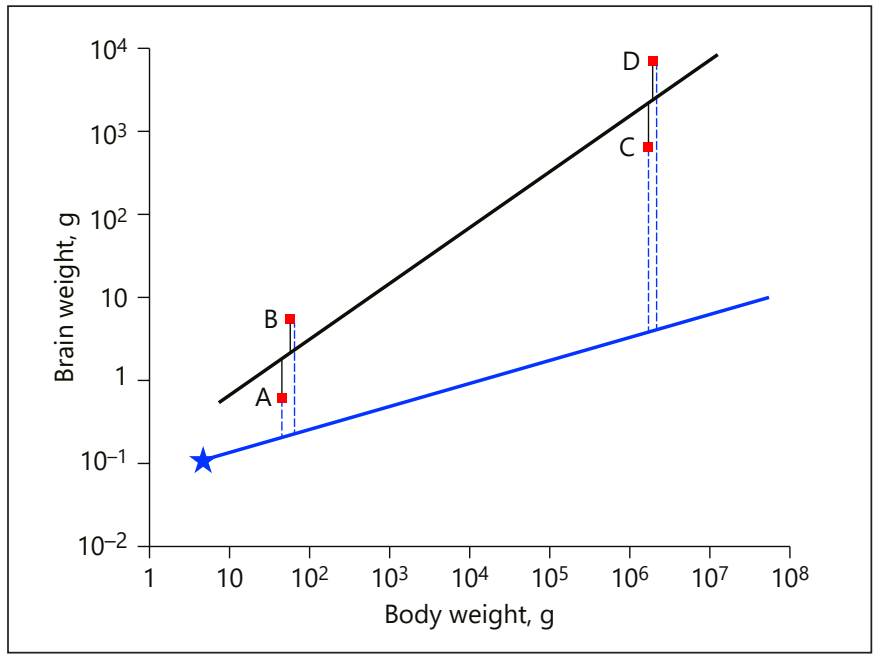

Fig. 1. Illustration of the difference between EQ and estimates of cognitive brain size. The steep black line gives the Jerison curve for the average mammalian brain and the shallow blue line the curve for the estimate of the somatic brain, which was anchored using the smallest-brained mammal S. minutus (star). Four hypothetical species (i.e., squares A-D) are included to illustrate the different measures. The black vertical lines are the residuals from the Jerison line; EQ is the ratio of the value for a given point and the value of the corresponding point on the black line. The blue dashed lines are the estimates of the cognitive brain size.

$\geq 0.25$. However, Lande [1985] suggested that the genetic correlation between brain and body size is lower in natural species. Indeed, Grabowski [2016] estimated it for samples of 5 primate species taken from the wild and found an average value of 0.254 , which should be somewhat lower than the true slope, given the error in body mass used to estimate genetic correlation between brain 
Fig. 2. Changes in cognitive brain size (a) and EQ (b) during hominin evolution. The dates are the median observed age (Ma before present). Data are based on Grabowski [2016].

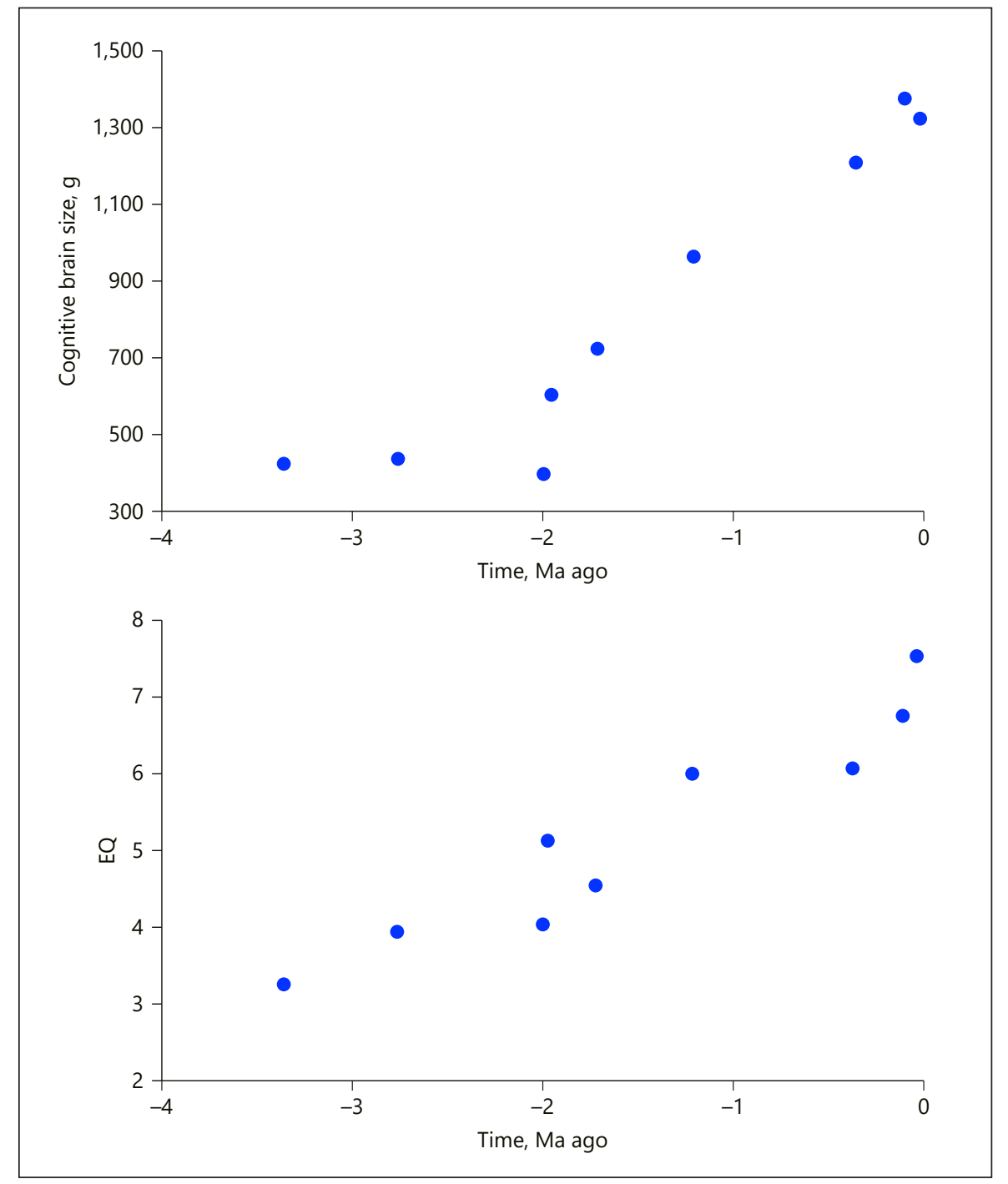

and body. Thus, our estimate of 0.27 for the slope of cognitive equivalence is quite consistent with these estimates of genetic correlation.

We therefore used a line of $\mathrm{P}^{0.27}$ (where $\mathrm{P}$ is body size) as the estimate of the somatic brain. For estimates of a species' cognitive brain we need to anchor the line (Fig. 1). To achieve positive values for all species in our sample, we took the presumably smallest-brained mammal, i.e., $S$. minutus, and forced the curve through its values (average body mass $5 \mathrm{~g}$ and average brain mass $0.1 \mathrm{~g}$ ) [Bauchot and Stephan, 1966]. This leads to the minimum estimated size of the somatic brain as $\mathrm{E}_{\mathrm{s}}=0.065 \mathrm{xP}^{0.27}$, and thus the following estimate of the cognitive brain size: $\mathrm{E}_{\mathrm{c}}=\mathrm{E}-\mathrm{E}_{\mathrm{s}}$, where $\mathrm{E}$ is the species' actual brain size. Figure 1 illustrates the procedure.
The most important result of the cognitive validation analyses was that the alternative measures, including body size, all perform better than the EQ. The EQ is the ratio of observed to expected brain size and, in Figure 1, the antilog of the residual from the Jerison line. Thus, a very small mammal could have the same ratio as a very large mammal (as do species B and D in Fig. 1) even if they differ dramatically in the absolute size of the estimated cognitive brain (the antilog of the blue dashed vertical lines). Under the EQ, the estimated order of overall cognitive performance would increase as follows: $\mathrm{A}=\mathrm{C}$ $<\mathrm{B}=\mathrm{D}$. Under the cognitive brain estimate deployed here it would be: $\mathrm{A}<\mathrm{B}<\mathrm{C}<\mathrm{D}$, i.e., the same order as in the absolute brain size or (in this example) the absolute body size of the species. 
The close similarity in how well the various non-EQ measures predicted actual estimates of overall cognitive abilities is not surprising. Absolute brain size is often a reasonable predictor of specific cognitive abilities in studies that did not use the measure based on cognitive equivalence [Benson-Amran et al., 2013; MacLean et al., 2014; Horschler et al., 2019]. The 2 measures are closely correlated and also of course show strong correlations with body size. Indeed, also the neocortex ratio remains highly correlated with body size [e.g., Stout, 2018]. Although we did not include the neocortex ratio in this study, in the data set used by Dunbar [1992], which uses averages for genera and sexes, the Spearman rank correlation between the 2 is 0.871 ( $n=38, p<0.001$; nonlinear relationship). Thus, the discrepancies among the various alternative approaches (absolute brain size, cognitive equivalence, and neocortex ratio) are quite modest, and in practice we will often not have the resolution needed to decide which of these is the best due to the high noise in the estimates of cognitive ability.

Based on these highly similar results, one could therefore argue that it is both most parsimonious and most convenient to use absolute brain size from now on, as has been proposed before. Nonetheless, we would suggest that the modest correction for body size evident in the estimate of the cognitive brain is conceptually necessary. First, the cognitive abilities of extremely large animals would almost certainly be overestimated. Second, and most importantly, sex differences in cognitive abilities within dimorphic species would otherwise be inferred which may well not exist. We assumed no sex differences in intelligence for nonhuman primates, consistent with the results of the admittedly noisy cognitive tests in various species. But the far more sophisticated data available for humans also support this conclusion: there is only a tiny (if any) gender difference in intelligence in humans [Irwing and Lynn, 2005] despite a clear gender difference in brain size [e.g., Martin, 1986] (mean: 1,498 mL for males and 1,326 $\mathrm{mL}$ for females). We therefore prefer the estimate based on cognitive equivalence.

Obviously, this approach reduces the effect of body size on brain size much more than has traditionally been considered necessary. It is not clear why this is. First, this conclusion may be peculiar to primates, with their large brains for mammals and thus large neocortices [Finlay et al., 2001]. Where cognitive functions take up a large proportion of the overall brain size, the relative importance of the somatic functions must become concomitantly smaller. If so, we expect the slope to be lower for other mammalian orders. Second, the size of the somatic brain may be small overall because neurons in many brain regions can be involved in multiple networks simultaneously, thus blurring the distinction between somatic and cognitive functions.

The analysis presented here is of course preliminary. It is based on primates only, and on a limited number of species at that, and could not establish whether species differences in slopes were true or merely reflected error. Future work should include all mammals with nontrivial dimorphism in body size, while ensuring the use of fully adult, wild specimens, with values not affected by advanced pregnancy or confounded by seasonal variation in adiposity. Along with work on other vertebrates, this work may find that slopes vary among lineages, and generate and test hypotheses on its causes.

Nevertheless, this study confirms that the effect of body size on the size of the somatic brain (the 2-point slope) varies considerably between mammals (and presumably birds) [Tsuboi et al., 2018] on the one hand and ectothermic vertebrates (fishes, amphibians, and reptiles) on the other hand. The latter vary between 0.4 and 0.5 within species [Tsuboi et al., 2018]. For fishes, Triki et al. [2021] obtained a mean intraspecific slope of 0.49 that is independent of body size variation. Furthermore, Triki et al. [2021] studied one fish species, i.e., the cleaner fish Labroides dimidiatus in more detail. Individual performance in various cognitive tasks was not correlated with body size, so that the intraspecific brain-body slope of 0.53 of this species indeed represents the slope of cognitive equivalence. The causes of this major difference between intraspecific slopes in endotherms versus ectotherm vertebrates remain unexplained.

It might be objected that comparing distantly related taxa on their cognitive performance based on brain measures only is not advised given the known differences in neuron densities in the brains of different clades [Herculano-Houzel, 2017] and the effect on brain size of highly divergent bauplans. We fully endorse this view. Comparing cognitive brain estimates among distantly related lineages may not be very revealing, and we should be very careful. Nonetheless, this restriction does not argue in favor of reviving the EQ approach.

\section{Applications: An Example}

Although a thorough application of the new method is beyond the scope of this paper, we can get a first impression by comparing the cognitive brain estimate with those of Jerison's [1973] EQ for extinct hominins thought to be in the main lineage contributing representatives that formed our own species (thus excluding the robust 
australopithecines and both Homo floresiensis and $\mathrm{H}$. naledi). Because the new measure produces cognitive brain size estimates that are rather close to overall brain size, the 2 techniques yield a rather different picture (Fig. 2). Jerison's [1973] EQ suggests a long period of a continuing, gradual increase in cognitive abilities from $4 \mathrm{Ma}$ until roughly $300 \mathrm{ka}$, followed by a sudden uptick. The alternative EQ measure proposed by Grabowski et al. [2016], which is based on a somewhat shallower slope, gives a similar picture, but without the sudden uptick in the last $300 \mathrm{ka}$ (see their Fig. 2). The cognitive equivalence measure, in contrast, suggests cognitive stasis between 4 and $2 \mathrm{Ma}$, after which cognitive abilities steadily increased.

It can be argued that the general pattern suggested by the cognitive equivalence measure is closer to the current understanding. Thus, the material culture of hominins did not exceed that of the extant great apes [Wynn et al., 2011] until the diversity and complexity of hominin material culture began in earnest around $2 \mathrm{Ma}$ [e.g., Stout, 2011], and there is no great increase in relative brain size over time within the human species [Schoenemann, 2013; Grabowski et al., 2016]. However, the paucity of material and the problems inherent in estimating body size from incomplete remains advise caution, and this alternative analysis should serve more to question the EQ-based interpretation than to support a specific alternative.

\section{Implications}

Comparative tests of adaptive hypotheses to explain brain size evolution in primates sometimes reach incompatible conclusions [Wartel et al., 2019], as do those on other mammals or birds [Healy and Rowe, 2007]. Among the many reasons for this impasse, one potentially prominent aspect is generally overlooked [Rogell et al., 2019]. These tests invariably include body mass as a control variable and then try to explain the remaining variation in brain size, in effect letting the pattern of covariation among the variables decide the allometric slope. In practice, therefore, the high correlation between body size and the other potential explanatory variables may affect the outcome and interpretation of the results [Stout, 2018], depending on which set of potential predictors are included in the analysis.

Thus, when we have a validated estimate of the size of the cognitive brain we can use this residual measure as the response variable, and so should get a fairer evaluation of the hypotheses trying to explain brain size evolution. In the end, this may be the most valuable contribution of the kind of analysis undertaken in this paper.

\section{Acknowledgment}

We thank Tsuboi Masahito for comments on an earlier version of this paper. C.P.S. thanks the Eugene Dubois Foundation for supporting his stay in Maastricht, The Netherlands, which inspired him to take up the quest again.

\section{Statement of Ethics}

Data were compiled from published studies.

\section{Conflict of Interest Statement}

The authors declare that they have no conflict of interests.

\section{Funding Sources}

This work was supported by the Swiss National Science Foundation (grant No.310030B_173334/1 to R.B. and P2NEP3_188240 to Z.T.).

\section{Author Contributions}

C.P.S. provided the conceptual basis, and R.B. and Z.T. added major discussion. S.A.H. provided data. S.A.H. and C.P.S. did the analyses. All of the authors wrote the final version. A preprint version of this article is available on bioRxiv [van Schaik et al., 2021].

\section{Data Availability Statement}

The compiled data for this study is accessible at the public repository Figshare (https://doi.org/10.6084/m9.figshare.14346959).

\section{References}

Alba DM. Cognitive inferences in fossil apes (Primates, Hominoidea): does encephalization reflect intelligence? J Anthropol Sci. 2010;88:11-48.

Alroy J. Cope's rule and the dynamics of body mass evolution in North American fossil mammals. Science. 1998 May;280(5364):7314.

Amici F, Barney B, Johnson VE, Call J, Aureli F. A modular mind? A test using individual data from seven primate species. PLoS One. 2012;7(12):e51918.

Arden R, Adams MJ. A general intelligence factor in dogs. Intelligence. 2016;55:79-85.

Armstrong E. Relative brain size and metabolism in mammals. Science. 1983 Jun;220(4603):1302-4.

Ashton BJ, Ridley AR, Edwards EK, Thornton A. Cognitive performance is linked to group size and affects fitness in Australian magpies. Nature. 2018 Feb;554(7692):364-7.

van Schaik/Triki/Bshary/Heldstab 
Barton RA. Primate brain evolution: integrating comparative, neurophysiological, and ethological data. Evol Anthropol. 2006;15(6):22436.

Barton RA, Harvey PH. Mosaic evolution of brain structure in mammals. Nature. 2000 Jun;405(6790):1055-8.

Bauchot R, Stephan H. Donnees nouvelles sur l'encephalisation des insectivores et des prosimiens. Mammalia. 1966;30(1):160-96.

Benoit J, Legendre LJ, Tabuce R, Obada T, Mararescul V, Manger P. Brain evolution in Proboscidea (Mammalia, Afrotheria) across the Cenozoic. Sci Rep. 2019 Jun;9(1):9323.

Benson-Amram S, Weldele ML, Holekamp KE. A comparison of innovative problem-solving abilities between wild and captive spotted hyaenas, Crocuta crocuta. Anim Behav. 2013;85(2):349-56.

Boddy AM, McGowen MR, Sherwood CC, Grossman LI, Goodman M, Wildman DE. Comparative analysis of encephalization in mammals reveals relaxed constraints on anthropoid primate and cetacean brain scaling. J Evol Biol. 2012 May;25(5):981-94.

Bronson RT. Brain weight-body weight scaling in breeds of dogs and cats. Brain Behav Evol. 1979;16(3):227-36.

Burkart JM, Schubiger MN, van Schaik CP. The evolution of general intelligence. Behav Brain Sci. 2017 Jan;40:e195.

Damerius LA, Forss SI, Kosonen ZK, Willems EP, Burkart JM, Call J, et al. Orientation toward humans predicts cognitive performance in orang-utans. Sci Rep. 2017 Jan;7(1):40052

Deacon TW. Rethinking mammalian brain evolution. Am Zool. 1990;30(3):629-705.

Deaner RO, Isler K, Burkart J, van Schaik C. Overall brain size, and not encephalization quotient, best predicts cognitive ability across non-human primates. Brain Behav Evol. 2007;70(2):115-24.

Deaner RO, Nunn CL, van Schaik CP. Comparative tests of primate cognition: different scaling methods produce different results. Brain Behav Evol. 2000 Jan;55(1):44-52.

Deaner RO, van Schaik CP, Johnson V. Do some taxa have better domain-general cognition than others? A meta-analysis of nonhuman primate studies. Evol Psychol. 2006;4(1):14996.

Dechmann DK, LaPoint S, Dullin C, Hertel M, Taylor JR, Zub K, et al. Profound seasonal shrinking and regrowth of the ossified braincase in phylogenetically distant mammals with similar life histories. Sci Rep. 2017 Feb;7(1):42443.

Dunbar RI. Neocortex size as a constraint on group size in primates. J Hum Evol. 1992;20(6):469-93.

Finlay BL, Darlington RB. Linked regularities in the development and evolution of mammalian brains. Science. 1995 Jun;268(5217):157884.

Finlay BL, Darlington RB, Nicastro N. Developmental structure in brain evolution. Behav Brain Sci. 2001 Apr;24(2):263-78.
Garamszegi LZ, Eens M. The evolution of hippocampus volume and brain size in relation to food hoarding in birds. Ecol Lett. 2004;7(12):1216-24.

Garamszegi LZ, Eens M, Erritzøe J, Møller AP. Sperm competition and sexually size dimorphic brains in birds. Proc Biol Sci. 2005 Jan;272(1559):159-66.

Gibson KR, Rumbaugh D, Baren M. Bigger is better: Primate brain size in relation to cognition. In: Falk D, Gibson KR, editors. Evolutionary anatomy of the primate cerebral cortex. Cambridge: Cambridge University Press; 2001. p. 79-97.

Grabowski M. Bigger brains led to bigger bodies? The correlated evolution of human brain and body size. Curr Anthropol. 2016;57(2):17496.

Grabowski M, Voje KL, Hansen TF. Evolutionary modeling and correcting for observation error support a 3/5 brain-body allometry for primates. J Hum Evol. 2016 May;94:106-16.

Halley AC, Deacon TW. The developmental basis of evolutionary trends in primate encephalization. Evolution of Nervous Systems. 2017;3:149-62.

Healy SD, Krebs JR. Food storing and the hippocampus in Paridae. Brain Behav Evol. 1996;47(4):195-9.

Healy SD, Rowe C. A critique of comparative studies of brain size. Proc Biol Sci. 2007 Feb;274(1609):453-64.

Heldstab SA, Isler K, Burkart JM, van Schaik CP. Allomaternal care, brains and fertility in mammals: who cares matters. Behav Ecol Sociobiol. 2019;73(6):71.

Heldstab SA, Isler K, van Schaik CP. Hibernation constrains brain size evolution in mammals. J Evol Biol. 2018 Oct;31(10):1582-8.

Heldstab SA, van Schaik CP, Isler K. Being fat and smart: A comparative analysis of the fat-brain trade-off in mammals. J Hum Evol. 2016 Nov; 100:25-34.

Hemmer H. Beitrag zur Erfassung der progressiven Cephalization bei Primaten. In: Biegert J, Leutenegger W, editors. Proc Third Intl Congr Primatol, Vol 1. Basel: S. Karger; 1971. pp. 99-107.

Herculano-Houzel S. Numbers of neurons as biological correlates of cognitive capability. Curr Opin Behav Sci. 2017;16:1-7.

Hopkins WD, Russell JL, Schaeffer J. Chimpanzee intelligence is heritable. Curr Biol. 2014 Jul;24(14):1649-52.

Horschler DJ, Hare B, Call J, Kaminski J, Miklósi Á, MacLean EL. Absolute brain size predicts dog breed differences in executive function. Anim Cogn. 2019 Mar;22(2):187-98.

Irwing P, Lynn R. Sex differences in means and variability on the progressive matrices in university students: a meta-analysis. Br J Psychol. 2005 Nov;96(Pt 4):505-24.

Isler K, Christopher Kirk E, Miller JM, Albrecht GA, Gelvin BR, Martin RD. Endocranial volumes of primate species: scaling analyses using a comprehensive and reliable data set. J Hum Evol. 2008 Dec;55(6):967-78.
Jerison HJ. Evolution of brain and intelligence. New York: Academic Press; 1973.

Kotrschal A, Räsänen K, Kristjánsson BK, Senn M, Kolm N. Extreme sexual brain size dimorphism in sticklebacks: a consequence of the cognitive challenges of sex and parenting? PLoS One. 2012;7(1):e30055.

Krompecher S, Lipák J. A simple method to determine cerebralization. J Comp Neurol. 1966;127:113-20.

Lande R. Quantitative genetic analysis of multivariate evolution, applied to brain: body size allometry. $\quad$ Evolution. 1979 Mar;33(1Part2):402-16.

Lande R. Genetic and evolutionary aspects of allometry. In: Jungers WL, editor. Size and scaling in primate biology. New York: Plenum Press; 1985. p. 21-32.

Leigh SR. Relations between captive and noncaptive weights in anthropoid primates. Zoo Biol. 1994;13(1):21-43.

Lindenfors P, Nunn CL, Barton RA. Primate brain architecture and selection in relation to sex. BMC Biol. 2007 May;5(1):20.

MacLean EL, Hare B, Nunn CL, Addessi E, Amici F, Anderson RC, et al. The evolution of selfcontrol. Proc Natl Acad Sci USA. 2014 May;111(20):E2140-8.

Martin RD. Relative brain size and basal metabolic rate in terrestrial vertebrates. Nature. 1981 Sep;293(5827):57-60.

Martin RD. Ontogenetic and phylogenetic aspects of human brain size. In: Sakka M, editor. Définition et origines de l'homme. Paris: Éditions du CNRS; 1986. p. 325-41.

Martin RD, Harvey PH. Brain size allometry: Ontogeny and phylogeny. In: Jungers WL, editor. Size and scaling in primate biology. New York: Plenum Press; 1985. p. 147-73.

Pagel MD. A method for the analysis of comparative data. J Theor Biol. 1992;156(4):431-42.

Pagel MD, Harvey PH. Taxonomic differences in the scaling of brain on body weight among mammals. Science. 1989 Jun;244(4912):158993.

Park HJ, Friston K. Structural and functional brain networks: from connections to cognition. Science. 2013 Nov;342(6158):1238411.

Passingham RE. The Human Primate. San Francisco: Freeman; 1982.

Pilbeam D, Gould SJ. Size and scaling in human evolution. Science. 1974 Dec;186(4167):892901

Portmann A. Etudes sur la cérébralisation chez les oiseaux. Alauda. 1946;14:2-20.

Portmann A. Etudes sur la cérébralisation chez les oiseaux. Alauda. 1947;15:1-15.

Reader SM, Hager Y, Laland KN. The evolution of primate general and cultural intelligence. Philos Trans R Soc Lond B Biol Sci. 2011 Apr;366(1567):1017-27.

Rensch B. Gedächtnis, Begriffsbildung und Planhandlungen bei Tieren. Hamburg: Paul Parey; 1973.

Riska B, Atchley WR. Genetics of growth predict patterns of brain-size evolution. Science. 1985 Aug;229(4714):668-71. 
Rogell B, Dowling DK, Husby A. Controlling for body size leads to inferential biases in the biological sciences. Evol Lett. 2019 Dec;4(1):7382.

Röhrs M. Cephalisation bei Caniden. J Zool Syst Evol Res. 1986;24(4):300-7.

Rowe TB, Macrini TE, Luo ZX. Fossil evidence on origin of the mammalian brain. Science. 2011 May;332(6032):955-7.

Rumbaugh DM, Savage-Rumbaugh ES, Washburn DA. Toward a new outlook on primate learning and behavior: complex learning and emergent processes in comparative perspective. Jpn Psychol Res. 1996 Sep;38(3):113-25.

Schoenemann PT. Brain size scaling and body composition in mammals. Brain Behav Evol. 2004;63(1):47-60.

Schoenemann TP. Hominid brain evolution. In: Begun DR, editor. A companion to paleoanthropology. Hoboken: Blackwell; 2013. p. 136-64.

Shettleworth SJ. Cognition, evolution, and behavior. 2nd ed. New York: Oxford University Press; 2010.
Stephan H. Methodische Studien über den quantitativen Vergleich architektonischer Struktureinheiten des Gehirns. Z Wiss Zool. 1960;164:143-72.

Sterck EH, Zijlmans DG, de Vries H, van den Berg LM, van Schaik CP, Langermans JA. Determining overweight and underweight with a new weight-for-height index in captive group-housed macaques. Am J Primatol. 2019 Jul;81(6):e22996.

Stout D. Stone toolmaking and the evolution of human culture and cognition. Philos Trans $\mathrm{R}$ SocLond B BiolSci.2011 Apr;366(1567):1050-

Stout D. Human brain evolution: history or science. In: Schwartz JH, editor. Rethinking Human Evolution. Cambridge (MA): MIT Press; 2018. pp. 297-317.

Striedter GF. Principles of Brain Evolution. Sunderland (MA): Sinauer Associates, Inc.; 2005.

Timmermans S, Lefebvre L, Boire D, Basu P. Relative size of the hyperstriatum ventrale is the best predictor of feeding innovation rate in birds. Brain Behav Evol. 2000 Oct;56(4):196203.
Triki Z, Aellen M, Schaik CV, Bshary R. Relative brain size and cognitive equivalence in fishes. 2021. Available from: https://doi.org/https:// doi.org/10.1101/2021.02.09.430417.

Tsuboi M, van der Bijl W, Kopperud BT, Erritzøe J, Voje KL, Kotrschal A, et al. Breakdown of brain-body allometry and the encephalization of birds and mammals. Nat Ecol Evol. 2018 Sep;2(9):1492-500.

van Schaik CP, Triki Z, Bshary R, Heldstab SA. A farewell to EQ: A new brain size measure for comparative primate cognition. 2021. Available from: https://doi.org/https://doi. org/10.1101/2021.02.15.431238.

Wartel A, Lindenfors P, Lind J. Whatever you want: inconsistent results are the rule, not the exception, in the study of primate brain evolution. PLoS One. 2019 Jul;14(7):e0218655.

Wells JC. The evolutionary biology of human body fat: Thrift and control. Cambridge: Cambridge University Press; 2010.

Wynn T, Hernandez-Aguilar RA, Marchant LF, McGrew WC. "An ape's view of the Oldowan” revisited. Evol Anthropol. 2011 SepOct;20(5):181-97. 